\title{
The short-term effect of COVID-19 pandemic on disability, pain intensity, psychological status, and exercise habits in patients with chronic pain
}

\author{
Aki Fujiwara ${ }^{1}\left({ }^{10} \cdot K^{2}\right.$ isuke Watanabe ${ }^{1} \cdot$ Mitsuru Ida $^{1} \cdot$ Hideaki Kawanishi $^{1} \cdot$ Katsuhiro Kimoto $^{1} \cdot$ Kie Yoshimura $^{1}$. \\ Kozue Shinohara ${ }^{1} \cdot$ Masahiko Kawaguchi $^{1}$
}

Received: 28 June 2021 / Accepted: 21 August 2021 / Published online: 25 August 2021

(c) Japanese Society of Anesthesiologists 2021

\begin{abstract}
Purpose The coronavirus disease 2019 (COVID-19) pandemic has affected the lives of people worldwide. The first declaration of a state of emergency in Japan, based on the Act on Special Measures for the Prevention and Control of the Novel Coronavirus, was issued from 16 April 2020 to 14 May 2020 to reduce person-to-person contact. Restrictions on going out, participating in community activities, and visiting hospitals were in place. This study investigates the short-term effects of the COVID-19 pandemic on patients with chronic pain.

Methods This study included outpatients with chronic pain undergoing treatment at the Pain Center of Nara Medical University Hospital. The patients had completed questionnaires for a disability during the study period, from 1 July to 30 September 2019 (baseline), 1 October to 31 December 2019 (pre-pandemic), and 1 July to 30 September 2020 (during the pandemic). The questionnaire covered changes in disability, pain intensity, health-related quality of life (QOL), anxiety, depression, catastrophic thinking, and the presence/absence of exercise habits at baseline, pre-pandemic, and during the pandemic.

Results Of the 245 eligible patients, there was no significant disability difference between baseline, pre-pandemic, and during the pandemic $(p=0.14)$. Similarly, pain intensity, health-related QOL, anxiety, depression, and the presence/absence of exercise habits did not significantly differ between baseline, pre-pandemic, and during the pandemic either. The current study observed significant differences in terms of catastrophic thinking $(p=0.02)$.

Conclusion The effects of the COVID-19 pandemic on patients with chronic pain were not apparent in the short-term. Clinical trail registration: UMIN000043174
\end{abstract}

Keywords COVID-19 pandemic $\cdot$ Chronic pain $\cdot$ Disability $\cdot$ NRS $\cdot$ Exercise habit

\section{Introduction}

The global spread of the coronavirus disease 2019 (COVID19) has prompted several countries to reduce person-to-person contact. Telemedicine-i.e., remote medical care-was recommended to patients with relatively stable conditions to prevent the spread of the infection and secure medical resources [1]. Also, patients refrained from visiting hospitals to avoid the risk of infection [2]. Patients with chronic pain received the following recommendations: suspend any

Aki Fujiwara

K119682@naramed-u.ac.jp

1 Department of Anesthesiology, Nara Medical University, 840 Shijo-cho, Kashihara, Nara 634-8522, Japan elective in-person patient visits, and undergo no elective pain procedures, barring specific semi-urgent procedures [1]. The first COVID-19 infection in Japan was confirmed on 16 January 2020, and it gradually spread nationwide. On 7 April 2020, the Japanese government issued a declaration of emergency in seven prefectures based on the Act on Special Measures for the Prevention and Control of the Novel Coronavirus.

Furthermore, the Japanese government extended the state of emergency to all prefectures on 16 April 2020. There were restrictions on going out, consequently reducing physical exercise, walking, and light exercise opportunities. It was lifted on 14 May 2020 in our prefecture, then, on 25 May 2020 nationwide. However, even after that, our lifestyle was not restored. People refrained to go out, meet friends, and participate local events to avoid the risk of infection. 
In our prefecture, inpatient treatment was limited only for emergency or deadly disease cases. Regarding outpatient treatment, we did not ask patients to reduce hospital visits and there were no restriction on pain management procedure same as the baseline. However, several home-visit rehabilitation and outpatient rehabilitation were closed in our area. We recommended continuing exercise habit such as walking for $15 \mathrm{~min}$ daily to the patients during the pandemic. There were 474 patients who were infected with COVID-19 virus from 1 July 2020 to 30 September 2020 in our prefecture. Number of infected people per million population was 119. Yamada et al. conducted an online survey of 1600 community-dwelling individuals $\geq 65$ years and lived in urban area in Japan [3]. They observed a significant decrease in the total duration of physical activity in April 2020 compared with January 2020. Notably, the COVID-19 pandemic would have negative physical and mental effects on patients with chronic pain, reducing exercise opportunities and increasing loneliness. However, the multidimensional evaluation, such as the 12-item World Health Organization Disability Assessment Schedule 2.0 (WHODAS 2.0), a disability assessment tool that includes cognition, mobility, self-care, getting along, life activities, and participation, has not been investigated. Moreover, some measures need to be taken if these patients have been adversely affected. Therefore, this study aimed to investigate (1) the effects of COVID-19 pandemic on disability, pain intensity, health-related QOL, psychological conditions, and presence/absence exercise habits in patients with chronic pain and (2) the effect of the COVID-19 pandemic on each group, classified depending on disability severity.

\section{Methods}

The current study evaluated data from "The Prevalence and Associated Factors of Functional Disability in Patients with Chronic Pain (UMIN000035149)" retrospectively. The Institutional Review Board of Nara Medical University approved this study (Approval No. 2955, 1 April 2021). All patients provided written informed consent to participate in "The Prevalence and Associated Factors of Functional Disability in Patients with Chronic Pain" study. In addition, patients eligible for this study were allowed to opt out on the Department of Anesthesiology of Nara Medical University website. This study was registered in the University Hospital Medical Information Network (UMIN) Clinical Trial Registry (UMIN000043174).

\section{Patient selection}

Patients ( $\geq 50$-years old) with chronic pain receiving treatment at the Pain Center of Nara Medical University Hospital, those who completed all questionnaires, including the 12-item (WHODAS 2.0)—a disability assessment tool-in the entire period, as shown below, were included in this study. The condition of the patients with acute pain may change over time. To select patients whose condition, including pain intensity, was stable, patients with chronic pain were defined as those receiving pain treatment for $\geq 90$ days at the pain center of the current study before baseline; these patients were included in this study. Patients who could not complete the questionnaire or had no pain at baseline (Numerical Rating Scale $[\mathrm{NRS}]=0$ ) were excluded. An evaluation was performed by comparing the baseline status (pre-pandemic) to the status during-COVID-19. This approach allowed identification of trends, i.e., whether each parameter before the COVID-19 pandemic was stable and to show whether there were improvements over time.

Baseline: between 1 July and 30 September 2019.

Pre-pandemic: between 1 October 2019 and 31 December 2019 before COVID-19 infections were detected in Japan.

During the pandemic: between 1 July and 30 September 2020, approximately three months after the first declaration of the state of emergency.

\section{Measurements}

The following data were collected: age, gender, the interval between visits to the pain center, disability (12-item WHODAS 2.0), pain intensity (NRS), health-related QOL (The Euro QOL 5-dimension 5-level [EQ5D5L]), anxiety (anxiety score of the Hospital Anxiety and Depression Scale [HADS]), depression (HADS depression score), catastrophic thinking (Pain Catastrophizing Scale [PCS]), and the presence or absence of exercise habits. According to the Ministry of Health, Labor and Welfare's "Individual Goals of Measures for the Elderly (Examples)," patients were considered to have an exercise habit if they could perform at least one of the following: exercise for $10 \mathrm{~min}$ a day, walking for $20 \mathrm{~min}$ a day, muscle training twice a week, and light sports thrice a week [4]. For the interval between visits, the day of the last visit up to 31 December 2019 was day 0 (visit 0). The number of days until the date of visit 3 was then counted retroactively and divided by 3 for pre-pandemic visits. For during- pandemic visits, the day of the first visit after 1 July 2020 was day 0 (visit 0 ), and the interval was the number of days until the day of the subsequent visit 3 divided by 3 . The intervals between the two periods, pre-pandemic and during the pandemic, were compared.

\section{Instruments}

The 12-item WHODAS 2.0 is a disability assessment tool with a recall period of 30 days. It consists of six domains (cognition-understanding and communicating; mobilitymoving and getting around; self-care—hygiene, dressing, 
eating, and staying alone; getting along-interacting with other people; life activities-domestic responsibilities, leisure, work, and school; and participation-joining in community activities) with 12 items. The patient has five choices for each item, and the score, depending on the choice, ranges from 0 (none) to 4 (extreme). According to the World Health Organization guideline, the scoring system based on the item-response theory was adopted, resulting in the range of $0-100(0=$ no disability; $100=$ full disability $)$ [5]. Disability severity is based on the calculated score: none (0-4), mild (5-24), moderate (25-49), severe (50-95), and complete (96-100) [6]. The clinically significant functional disability was defined as a 12-item WHODAS 2.0 score of $\geq 25$ [7]. Moreover, the minimum clinically important difference (MCID) is 8.

NRS is one of the most commonly used pain scales in medicine. It is commonly $0-10$ with 0 (no pain) and 10 (the worst pain imaginable."). The patients select an answer between 0 and 10 that fits best to their pain intensity.

The EQ5D5L is a standard instrument used to measure the QOL with five dimensions: mobility, self-care, usual activities, pain/discomfort, and anxiety/depression. Each dimension has five levels and defines 3125 health states. Moreover, it has a country-specific scoring system. These health states are expressed as a value from -0.025 to 1 (full health) [8]. The MCID is 0.1 for chronic pain [9].

The HADS is a self-assessment tool to evaluate anxiety and depression. It consists of 14 items equally divided into the anxiety (HADS Anxiety) and depression (HADS depression) subscales. Patients with a HADS Anxiety score of $\geq 11$ are considered to have anxiety. Moreover, patients with a HADS depression score of $\geq 11$ are considered to have depression [10].

The PCS consists of 13 items, each of which is rated on a scale of 0-4. Points are allocated according to the answer to each item. The level of catastrophic thinking is considered high if the total score is $\geq 30$ [11].

\section{Variables}

Disabilities in patients with chronic pain were assessed based on the 12-item WHODAS 2.0 scores at baseline, pre-pandemic, and during the pandemic. In addition, NRS, EQ5D5L, HADS, HADS depression, PCS, and the presence/ absence of exercise habits were evaluated.

A study investigating mental health and social interactions of older people with physical disabilities during the early months of the COVID-19 pandemic in England showed that older people with physical disabilities had more symptoms of depression and anxiety, greater loneliness, poorer life satisfaction, and lower purpose in life, quality of life, and sleep quality than people without a physical disability [12]. Thus, considering the possibility that the effect of the
COVID-19 pandemic may differ depending on the severity of the disability at baseline, patients were classified into four groups (none, mild, moderate, and severe + complete). The 12-item WHODAS 2.0 scores of 0-4, 5-24, 25-49, and 50-100 were defined as none, mild, moderate, and severe and complete [6]. Furthermore, the 12-item WHODAS2.0, NRS, EQ5D5L, anxiety, depression, PCS, and the presence/absence of exercise habits within each group were investigated.

\section{Statistical analyses}

The Friedman tests were performed for the 12-item WHODAS 2.0, NRS, EQ5D5L, anxiety, depression, and PCS. Moreover, Bonferroni's method made multiple comparison adjustments. Cochran's $Q$ test compared the presence/ absence of exercise habits. All data were analyzed using EZR version 3.1.1 (14 July 2010, Copyright (C) 2014, The R Foundation for Statistical Computing Platform: i386-w64-mingw32/i386) [13], and a $p$-value of $<0.05$ indicated statistical significance.

\section{Results}

Initially, 402 patients completed the questionnaires at baseline. Moreover, 27 and nine patients with a baseline visit duration of $<90$ days and NRS of 0 , respectively, were excluded. Of the 366 patients, 86 did not complete the questionnaire during the pre-pandemic period. Furthermore, 21 of the 280 remaining patients did not complete the questionnaire during the pandemic period. Of the 259 patients who completed the questionnaire in all three periods, 14 patients were regarded as incomplete responses because there were one or more blanks in their questionnaires, resulting in a final review of the 245 patients (Fig. 1). There were no patients who were suspected of being infected with COVID19 in this study. The primary disease for visiting our pain center was showed in Table 1.

Table 2 shows the background characteristics of the patients and the results. The interval between visits was significantly longer during the pandemic (median [interquartile range (IQR)]: 30.0 [19.0] and 42.0 [49.3], respectively; $p<0.001)$. Moreover, the 12 -item WHODAS 2.0 scores were not significantly different across the baseline, prepandemic, and during the pandemic (median [IQR]: 25.0 [31.3], 25.0 [31.3] and 25.0 [31.3], respectively; $p=0.14$ ). The domain scores of WHODAS 2.0 were not significantly different across the three periods. However, this results were just for reference purpose because we did not use the 36-item WHODAS 2.0 (Table 2). There were no significant differences in NRS, EQ5D5L, anxiety, and depression across the three periods. The null hypothesis that the PCS 


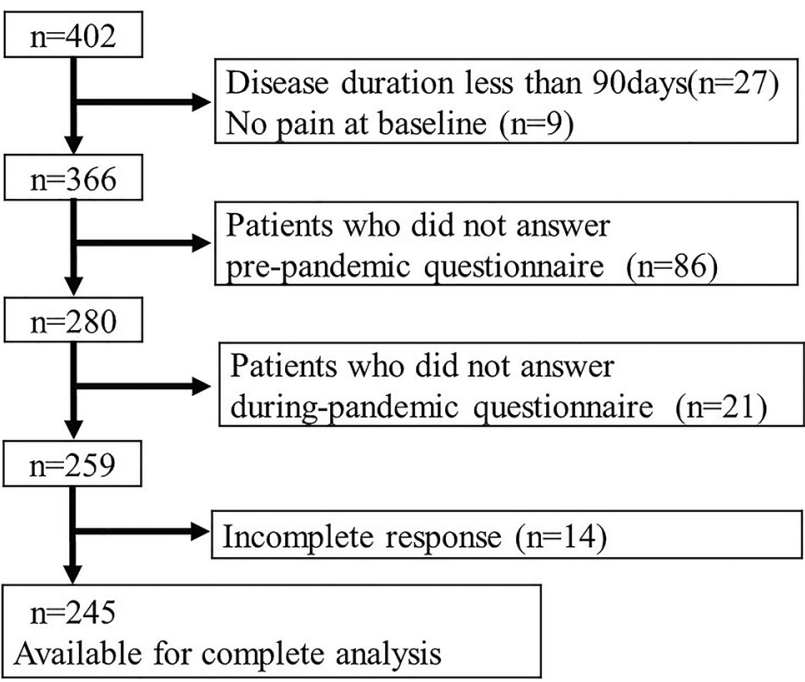

Fig. 1 Flow diagram of participation

for the baseline, pre-pandemic, and during the pandemic was identical was rejected $(p=0.02)$. However, the multiple comparison test could not detect any significant differences between the periods. Furthermore, there were no significant differences in the presence or absence of exercise habits.

According to the baseline classification by the severity of the disability, 39 patients were in the no disability group (12-item WHODAS 2.0, 0-4), 83 patients were in the mild disability group (12-item WHODAS 2.0, 5-24), 80 patients were in the moderate disability group (12-item WHODAS $2.0,25-49), 42$ patients were in the severe disability group (12-item WHODAS 2.0, 50-95), and one patient was in the complete disability group (12-item WHODAS 2.0, 96-100). One patient in the complete disability group was grouped along with the patients in the severe group and handled together as 43 patients in the severe + complete group. Table 3 shows the results for each group. The 12 -item WHODAS 2.0 scores in the group without disabilities was not the same in the three periods (median [IQR]: 0.0 [2.1], 2.1 [6.3], and 2.1 [3.1], respectively; $p=0.004$ ), and the multiple comparison test showed a significant difference between the baseline and pre-pandemic $(p=0.0013)$. In the severe + complete group, a significant difference in anxiety was noted across the three periods (median [IQR]: 10.0 [5.5], 8.0 [5.5], and 10.0 [7.0], respectively; $p=0.04$ ) and between baseline and the pre-pandemic $(p=0.03)$. Furthermore, there were no significant differences in the other variables across the three periods.

Table 1 The primary disease for visiting our pain center

\begin{tabular}{|c|c|c|c|c|c|c|}
\hline ICD10 & Disease & None $(n=39)$ & Mild $(n=83)$ & Moderate $(n=80)$ & $\begin{array}{l}\text { Severe and } \\
\text { complete } \\
(n=43)\end{array}$ & Total $(n=245)$ \\
\hline M47.86 & Lumbosacral spondylosis & $5(12.8)$ & $15(16.1)$ & $22(27.5)$ & $10(23.3)$ & $52(21.2)$ \\
\hline M48.06 & Lumbar spinal stenosis & $1(2.6)$ & $17(20.5)$ & $18(22.5)$ & $8(18.6)$ & $44(18.0)$ \\
\hline G53.0 & Postzoster neuralgia & $8(20.5)$ & $7(8.4)$ & $6(7.5)$ & $7(16.3)$ & $28(11.4)$ \\
\hline M47.82 & Cervical spondylosis & $3(7.7)$ & $8(9.6)$ & $9(11.3)$ & $1(2.3)$ & $21(8.6)$ \\
\hline G50.0 & Trigeminal neuralgia & $5(12.8)$ & $6(7.2)$ & $2(2.5)$ & $0(0)$ & $13(5.3)$ \\
\hline M51.1 & $\begin{array}{l}\text { Lumbar and other intervertebral disc disorders } \\
\text { with radiculopathy }\end{array}$ & $2(5.1)$ & $7(8.4)$ & $2(2.5)$ & $1(2.3)$ & $12(4.9)$ \\
\hline S32.0 & Fracture of lumbar vertebra & $0(0)$ & $1(1.2)$ & $4(5.0)$ & $3(7.0)$ & $8(3.3)$ \\
\hline G64 & Other disorders of peripheral nervous system & $1(2.6)$ & $5(6.0)$ & $2(2.5)$ & $0(0)$ & $8(3.3)$ \\
\hline M19.95 & Arthrosis, unspecified (Pelvic region and thigh) & $2(5.1)$ & $2(2.4)$ & $1(1.3)$ & $1(2.3)$ & $6(2.4)$ \\
\hline I73.9 & Peripheral vascular disease & $2(5.1)$ & $2(2.4)$ & $1(1.3)$ & $1(2.3)$ & $6(2.4)$ \\
\hline M89.0 & Algoneurodystrophy & $1(2.6)$ & $1(1.2)$ & $1(1.3)$ & $1(2.3)$ & $4(1.6)$ \\
\hline M75.0 & Adhesive capsulitis of shoulder & $2(5.1)$ & $2(2.4)$ & $0(0)$ & $0(0)$ & $4(1.6)$ \\
\hline M48.02 & Cervical spinal stenosis & $3(7.7)$ & $1(1.2)$ & $0(0)$ & $0(0)$ & $4(1.6)$ \\
\hline M17 & Gonarthrosis & $0(0)$ & $1(1.2)$ & $2(2.5)$ & $1(2.3)$ & $4(1.6)$ \\
\hline M43.1 & Spondylolisthesis & $0(0)$ & $1(1.2)$ & $1(1.3)$ & $2(4.7)$ & $4(1.6)$ \\
\hline others & Others & $4(10.3)$ & $7(8.4)$ & $9(11.3)$ & $7(16.3)$ & $27(11.0)$ \\
\hline Total & & $39(100)$ & $83(100)$ & $80(100)$ & $43(100)$ & $245(100)$ \\
\hline
\end{tabular}

Number (\%)

ICD International Classification of Diseases 
Table 2 Patient background characteristics and results

\begin{tabular}{llllc}
\hline & Baseline & Pre-pandemic & During the pandemic & $p$ value \\
\hline Age & $73.0[12.0]$ & & & \\
Female & $134(54.7)$ & & & \\
Duration of treatment before & $1133[1549]$ & & & \\
baseline assessment (days) & & & & \\
Interval of hospital visits & & $30.3[19.0]$ & $42.0[49.3]$ & 0.001 \\
12-item WHODAS 2.0 (total) & $25.0[31.3]$ & $25.0[31.3]$ & $25.0[31.3]$ & 0.14 \\
Cognition (Q3, Q6) & $1.0[3.0]$ & $1.0[3.0]$ & $1.0[3.0]$ & 0.58 \\
Mobility (Q1, Q7) & $3.0[4.0]$ & $3.0[5.0]$ & $3.0[4.0]$ & 0.72 \\
Self-care (Q8, Q9) & $1.0[2.0]$ & $0.0[2.0]$ & $0.0[2.0]$ & 0.97 \\
Getting along (Q10, Q11) & $0.0[2.0]$ & $0.0[2.0]$ & $0.0[2.0]$ & 0.08 \\
Life activities (Q2, Q12) & $2.0[3.0]$ & $2.0[3.0]$ & $2.0[3.0]$ & 0.42 \\
Participation (Q4, Q5) & $3.0[4.0]$ & $3.0[4.0]$ & $3.0[4.0]$ & 0.05 \\
NRS & $6.0[4.0]$ & $6.0[5.0]$ & $6.0[4.0]$ & 0.85 \\
EQ5D5L & $0.67[0.29]$ & $0.69[0.27]$ & $0.69[0.30]$ & 0.24 \\
Anxiety & $6.0[7.0]$ & $6.0[5.0]$ & $6.0[6.0]$ & 0.28 \\
Depression & $7.0[5.0]$ & $7.0[6.0]$ & $7.0[6.0]$ & 0.82 \\
PCS & $30.0[16.0]$ & $30.0[16.0]$ & $30.0[19.0]$ & 0.02 \\
Presence of exercise habits & $161(66.1)$ & $170(69.2)$ & $165(67.3)$ & 0.5 \\
Calisthenics & $96(39.2)$ & $87(35.5)$ & $97(39.6)$ & 0.35 \\
Walking & $99(40.4)$ & $103(42.0)$ & $101(41.2)$ & 0.86 \\
Working out & $66(26.9)$ & $70(28.6)$ & $73(29.8)$ & 0.69 \\
Light sports & $30(12.2)$ & $29(11.8)$ & $19(8.4)$ & 0.08 \\
\hline
\end{tabular}

Median [interquartile range] or Number (\%)

12-item WHODAS 2.0 the 12-item World Health Organization Disability Assessment Schedule 2.0, EQ5D5L Euro QOL 5dimmention5level, NRS Numerical Rating Scale, PCS Pain Catastrophizing Scale

\section{Discussion}

The COVID-19 pandemic situation has changed people's lifestyles. Restrictions exist in doing light exercise in the park and light sports (e.g., gateball), and sports gyms have been closed. Also, the use of daily services and outpatient rehabilitation have been restricted. The hypothesis was that the COVID-19 pandemic could exacerbate disability and QOL in patients with chronic pain because exercises, distractions, and relationships are important for maintaining daily life functions and QOL in patients with chronic pain [14]. However, this study results showed no significant changes across baseline, pre-pandemic, and during the pandemic regarding disability and pain intensity, health-related QOL, anxiety, depression, and presence/absence of exercise habits. Three possible causes for this may exist. First, the impacts may not have been apparent yet because assessment during the pandemic was performed only 3-5 months after the emergency declaration. In the future, physical and mental adverse effects (e.g., loss of exercise habits, increased pain, worsening disability, decreased health perception and QOL, anxiety and depression, and worsening of catastrophic thinking) may become more prominent if such a situation persists for a longer period. Second, the impact may have differed from place to place. Namely, the situation surrounding patients varied from region to region. Our prefecture was a suburban area, therefore, the impact of COVID-19 pandemic might be less than an urban area such as Tokyo. At early May 2020, the number of confirmed COVID-19 cases in Japan was less than 20,000 [15], while there were over 1.3 million and over 2,00,000 COVID-19 cases in the United States and United Kingdom, respectively [16]. Third, patients with chronic pain may be less susceptible to external factors (e.g., restrictions on going out). Some studies showed a stable disease trajectory in persistent pain intensity levels over time in chronic pain patients [17]. A study investigating the short-term effects of the COVID-19 pandemic in chronic pain patients seen at tertiary multidisciplinary pain centers in Germany showed that pain-related disability, health-related QOL, depression, and anxiety did not differ significantly from those observed before the COVID-19 pandemic [18]. They concluded that chronic pain disorder is a relatively stable disease that does not change significantly owing to external factors. They did not evaluate exercise habits. The current study evaluated the degree of disability, health-related QOL, pain intensity, anxiety, depression, catastrophic thinking, and the presence/absence of exercise habits. Unlike those of studies on healthy community-dwelling 
Table 3 The results for each group which was classified by severity of disability

\begin{tabular}{|c|c|c|c|c|}
\hline Disability & None $(n=39)$ & Mild $(n=83)$ & Moderate $(n=80)$ & $\begin{array}{l}\text { Severe and } \\
\text { complete } \\
(n=43)\end{array}$ \\
\hline Age & $69.0[9.5]$ & $75.0[10.5]$ & $72.0[14.3]$ & $75.0[14.5]$ \\
\hline Female & $15(38.5)$ & $40(48.2)$ & $50(62.5)$ & $29(67.4)$ \\
\hline \multicolumn{5}{|c|}{ Duration of treatment before baseline (days) } \\
\hline Baseline & $1436.0[1629.0]$ & $1139.0[1739.0]$ & $968.5[1409.5]$ & $1209.0[1330.0]$ \\
\hline \multicolumn{5}{|c|}{ Interval of hospital visits (days) } \\
\hline Pre-pandemic & $37.3[34.0]$ & $32.7[28.7]$ & $30.3[10.2]$ & $28.0[7.7]$ \\
\hline During the pandemic & $57.0[48.3]$ & $56.0[53.0]$ & $33.8[30.5]$ & $32.7[31.4]$ \\
\hline$p$ value & $<0.001$ & $<0.001$ & $<0.001$ & $<0.001$ \\
\hline \multicolumn{5}{|l|}{ 12-item WHODAS 2.0} \\
\hline Baseline & $0.0[2.1]$ & $12.5[8.3]$ & $35.4[10.4]$ & $58.3[13.5]$ \\
\hline Pre-pandemic & $2.1[6.3]$ & $16.7[14.6]$ & $34.4[14.6]$ & $58.3[20.8]$ \\
\hline During the pandemic & $2.1[3.1]$ & $14.6[16.7]$ & $32.3[15.1]$ & $60.4[27.1]$ \\
\hline$p$ value & $<0.01$ & 0.66 & 0.42 & 0.33 \\
\hline \multicolumn{5}{|l|}{ NRS } \\
\hline Baseline & $3.0[3.5]$ & $4.0[3.0]$ & $6.0[4.0]$ & $7.0[1.0]$ \\
\hline Pre-pandemic & $3.0[4.5]$ & $5.0[4.0]$ & $6.0[4.0]$ & $7.0[2.0]$ \\
\hline During the pandemic & $3.0[4.0]$ & $5.0[5.0]$ & $6.0[3.0]$ & $7.0[2.0]$ \\
\hline$p$ value & 0.78 & 0.44 & 0.64 & 0.28 \\
\hline \multicolumn{5}{|l|}{ EQ5D5L } \\
\hline Baseline & $0.89[0.07]$ & $0.78[0.18]$ & $0.60[0.16]$ & $0.38[0.31]$ \\
\hline Pre-pandemic & $0.89[0.08]$ & $0.78[0.16]$ & $0.64[0.16]$ & $0.39[0.28]$ \\
\hline During the pandemic & $0.89[0.04]$ & $0.78[0.19]$ & $0.61[0.23]$ & $0.36[0.27]$ \\
\hline$p$ value & 0.86 & 0.05 & 0.64 & 0.87 \\
\hline \multicolumn{5}{|l|}{ Anxiety } \\
\hline Baseline & $3.0[3.5]$ & $5.0[5.0]$ & $8.0[6.3]$ & $10.0[5.5]$ \\
\hline Pre-pandemic & $4.0[3.5]$ & $5.0[4.0]$ & $6.5[4.0]$ & $8.0[5.5]$ \\
\hline During the pandemic & $3.0[5.0]$ & $4.0[4.0]$ & $7.0[5.0]$ & $10.0[7.0]$ \\
\hline$p$ value & 0.33 & 0.08 & 0.89 & 0.04 \\
\hline \multicolumn{5}{|l|}{ Depression } \\
\hline Baseline & $3.0[3.5]$ & $6.0[5.0]$ & $8.0[4.0]$ & $10.0[5.0]$ \\
\hline Pre-pandemic & $4.0[4.0]$ & $6.0[6.0]$ & $7.0[4.0]$ & $10.0[5.5]$ \\
\hline During the pandemic & $4.0[6.5]$ & $5.0[4.5]$ & $7.0[5.0]$ & $11.0[5.5]$ \\
\hline$p$ value & 0.14 & 0.98 & 0.28 & 0.65 \\
\hline \multicolumn{5}{|c|}{ Pain Catastrophizing Scale } \\
\hline Baseline & $21.0[13.0]$ & $25.0[15.0]$ & $34.5[14.5]$ & $40.0[10.5]$ \\
\hline Pre-pandemic & $21.0[15.0]$ & $27.0[15.5]$ & $33.0[14.0]$ & $38.0[12.5]$ \\
\hline During the pandemic & $19.0[21.5]$ & $27.0[18.0]$ & $32.0[15.3]$ & $37.0[15.5]$ \\
\hline$p$ value & 0.28 & 0.96 & 0.14 & 0.07 \\
\hline \multicolumn{5}{|c|}{ Presence of exercise habits } \\
\hline Baseline & $30(76.9)$ & $59(71.1)$ & $53(66.3)$ & $20(46.5)$ \\
\hline Pre-pandemic & $30(76.9)$ & $65(78.3)$ & $56(70.0)$ & $19(44.2)$ \\
\hline During the pandemic & $28(71.8)$ & $61(73.5)$ & $53(66.3)$ & $23(53.5)$ \\
\hline$p$ value & 0.51 & 0.26 & 0.74 & 0.40 \\
\hline \multicolumn{5}{|l|}{ Presence of calisthenics } \\
\hline Baseline & $17(43.6)$ & $34(41.0)$ & $34.0(42.5)$ & $10(23.3)$ \\
\hline Pre-pandemic & $11(28.2)$ & $34(41.0)$ & $33.0(41.3)$ & $9(20.9)$ \\
\hline During the pandemic & $18(46.2)$ & $37.0(44.6)$ & $31(38.8)$ & $11(25.6)$ \\
\hline \multicolumn{5}{|l|}{ Presence of walking } \\
\hline Baseline & 17 (43.6) & $41(49.4)$ & $33(41.3)$ & 8 (18.6) \\
\hline
\end{tabular}


Table 3 (continued)

\begin{tabular}{lllll}
\hline Disability & None $(n=39)$ & Mild $(n=83)$ & Moderate $(n=80)$ & $\begin{array}{l}\text { Severe and } \\
\text { complete } \\
(n=43)\end{array}$ \\
\hline $\begin{array}{l}\text { Pre-pandemic } \\
\text { During the pandemic }\end{array}$ & $19(48.7)$ & $49(59.0)$ & $29(36.3)$ & $6(14.0)$ \\
Presence of working out & & $45(54.2)$ & $31(38.8)$ & $7(16.3)$ \\
Baseline & $10(25.6)$ & $25(30.1)$ & $22(27.5)$ & $9(20.9)$ \\
Pre-pandemic & $8(20.5)$ & $28(33.7)$ & $24(30.0)$ & $10(23.3)$ \\
During the pandemic & $11(28.2)$ & $21(25.3)$ & $25(31.3)$ & $16(37.2)$ \\
Presence of light sports & & & & $6(14.0)$ \\
Baseline & $7(17.9)$ & $9(20.8)$ & $8(10.0)$ & $1(2.3)$ \\
Pre-pandemic & $9(23.1)$ & $10(12.0)$ & $9(11.3)$ & $3(7.0)$ \\
During the pandemic & $5(12.8)$ & $7(8.4)$ & $4(5.0)$ & \\
\hline
\end{tabular}

Median [interquartile range] or Number (\%)

12-item WHODAS 2.0 the 12-item World Health Organization Disability Assessment Schedule 2.0, EQ5D5L Euro QOL 5dimension 5level, NRS Numerical Rating Scale

individuals [3], this study's results did not show significant changes in the presence/absence of exercise habits. Patients in chronic pain treatment are taught to exercise through physical activities (e.g., walking) [19]. Patients with preexisting pain may have had a habit of exercise that could be performed independently inside or around their home (e.g., physical exercise or walking) compared to healthy residents in the community. Regarding types of exercise, calisthenics and walking were much more common than working out and light sports in this study. Generally, calisthenics and walking did not require the use of public facilities. Therefore, they may have been less affected by the pandemic.

The null hypothesis that the median PCS was identical at baseline, pre-pandemic, and during the pandemic was rejected. However, the Bonferroni-adjusted multiple comparisons failed to detect any significant inter-period differences. Whether this is a treatment effect or the effect of the COVID-19 pandemic remains unclear.

In the analysis by severity, patients with more severe diseases were expected to experience further worsening in their disabilities. However, most factors were not significantly different in any group. In the group without disabilities, disabilities worsened significantly between the baseline and pre-pandemic periods, but the MCID of the 12-item WHODAS 2.0 score was 8 , suggesting that the change was not clinically significant. The COVID-19 pandemic's effect is unclear because no significant differences were observed pre-pandemic and during the pandemic. Although anxiety in the severe + complete group significantly improved in the pre-pandemic period than baseline, there were no significant differences between the pre-pandemic and during pandemic periods. For this finding, the impact of the COVID-19 pandemic may have offset the effect of clinical pain treatment because we did not restrict outpatient treatments during pandemic.
This study has several limitations. First, the study is limited to patients with chronic pain from a single institution. Thus, although the results of this study reflect the status of patients with chronic pain who continue receiving treatment at pain clinics, the status of patients being treated at other departments or patients who have not been treated remains unknown. Second, the impact of COVID-19 pandemic would differ from region to region. The patients in this study might have less impact than other countries. More studies examined the impact of COVID-19 in various countries are needed to draw a conclusion. Third, the current study only assessed short-term changes. Long-term investigations are needed to determine the true effects because the effects of the COVID-19 pandemic may become apparent over time. Fourth, we examined only existence of exercise habit, namely, we did not measure the amount of exercise. If we measured the amount of exercise using a pedometer or an activity monitor, the result might have changed.

\section{Conclusion}

The effect of the COVID-19 pandemic in patients with chronic pain was not apparent in the short-term. However, careful monitoring is required in the future because longterm effects are unknown.

Author contributions $\mathrm{AF}, \mathrm{KW}, \mathrm{MI}$, and MK contributed to the study conception and design. Material preparation and data collection were performed by AF, KW, KK, KY, KS, and HK. Data analysis was performed AF and MI. The first draft of the manuscript was written by AF. MK, KW and MI reviewed and edited the manuscript. All authors commented on previous version of the manuscript. All authors read and approved the final manuscript. 
Funding This study had no external funding in the preparation of this manuscript.

\section{Declarations}

Conflict of interest Each author certifies that he or she, or a member of his or her immediate family, has no commercial association (i.e., consultancies, stock ownership, equity interest, patent/licensing arrangements, etc.) that might pose a conflict of interest in connection with the submitted manuscript.

Consent for publication This manuscript has not been published elsewhere and are not under consideration for publication elsewhere.

\section{References}

1. Shanthanna H, Strand NH, Provenzano DA, Lobo CA, Eldabe S, Bhatia A, Wegener J, Curtis K, Cohen SP, Narouze S. Caring for patients with pain during the COVID-19 pandemic: consensus recommendations from an international expert panel. Anesthesia. 2020. https://doi.org/10.1111/anae.15076.

2. Mantica G, Riccardi N, Terrone C, Gratarola A. Non-COVID-19 visits to emergency departments during the pandemic: the impact of fear. Public Health. 2020;183:40-1.

3. Yamada M, Kimura Y, Ishiyama D, Otobe Y, Suzuki M, Koyama S, Kikuchi T, Kusumi H, Arai H. Effect of the COVID-19 epidemic on physical activity in community-dwelling older adults in Japan: a cross-sectional online survey. J Nutr Health Aging. 2020;24:948-50.

4. Ministry of Health, Labour and Welfare. Kenkou Nihon 21 [Ministry of Health, Labour and Welfare web site]. 2021. https://www. mhlw.go.jp/www1/topics/kenko21_11/b2.html\#A25. Accessed 21 Jan 2021.

5. Ustün TB, Chatterji S, Kostanjsek N, Rehm J, Kennedy C, EppingJordan J, Saxena S, von Korff M, Pull C, WHO/NIH Joint Project. Developing the World Health Organization Disability Assessment Schedule 2.0. Bull World Health Organ. 2010;88(815):23.

6. Ustün TB, Kostanjesek N, Chatterji S, Rehm J. Measuring Health and Disability: Manual for WHO Disability Assessment Schedule (12-item WHODAS 2.0). Geneva: World Health Organization. 2010. https://apps.who.int/iris/bitstream/handle/10665/43974/ 9789241547598_eng.pdf?sequence $=1 \&$ isAllowed $=y$. Accessed 21 Jan 2021.

7. Shulman MA, Myles PS, Chan MT, McIlroy DR, Wallace S, Ponsford J. Measurement of disability-free survival after surgery. Anesthesiology. 2015;122:524-36.
8. Herdman M, Gudex C, Lloyd A, Janssen M, Kind P, Parkin D, Bonsel G, Badia X. Development and preliminary testing of the new five-level version of EQ-5D (EQ-5D-5L). Qual Life Res. 2011;20:1727-36.

9. Alentado VJ, Caldwell S, Gould HP, Steinmetz MP, Benzel EC, Mroz TE. Independent predictors of a clinically significant improvement after lumbar fusion surgery. Spine J. 2017; 17:236-43.

10. Zigmond AS, Snaith RP. The hospital anxiety and depression scale. Acta Psychiatr Scand. 1983;67:361-70.

11. Sullivan MJ, Bishop SR, Pivik J. Pain catastrophizing scale: development and validation. Psychol Assess. 1995;7:524-32.

12. Steptoe A, Di Gessa G. Mental health and social interactions of ol mic: a longitudinal cohort study. Lancet Public Health. 2021;6:e365-73.

13. Kanda Y. Investigation of the freely available easy-to-use software 'EZR' for medical statistics. Bone Marrow Transplant. 2013;48:452-8. https://doi.org/10.1038/bmt.2012.244.

14. Malfliet A, Ickmans K, Huysmans E, Coppieters I, Willaert W, Bogaert WV, Rheel E, Bilterys T, Wilgen PV, Nijs J. Best evidence rehabilitation for chronic pain part 3; low back pain. J Clin Med. 2019. https://doi.org/10.3390/jcm8071063.

15. Hasegawa J, Arakaki T, Sekizawa A, Ikeda T, Ishiwata I, Kinoshita K. Current status of community-acquired infection of COVID-19 in delivery facilities in Japan. PLoS ONE. 2021;16(5):e0251434. https://doi.org/10.1371/journal.pone.0251434.

16. Lauxmann MA, Santucci NE, Autrán-Gómez M. The SARSCoV-2 coronavirus and the COVID-19 outbreak. Int Braz J Urol. 2020;46(suppl.1):6-18.

17. Glette M, Stiles TC, Borchgrevink PC, Landmark T. The natural course of chronic pain in a general population: stability and change in an eight-wave longitudinal study over four years (the HUNT pain study). J pain. 2020;21:689-99.

18. Lassen CL, Siam L, Degenhart A, Klier TW, Bundscherer A, Lindenberg N. Short-term impact of the COVID-19 pandemic on patients with a chronic pain disorder. Medicine. 2021;100(10):e25153. https://doi.org/10.1097/MD.0000000000 025153.

19. Geneen LJ, Martin DJ, Adams N, Clarke C, Dunbar M, Jones D, McNamee P, Schofield P, Smith BH. Effects of education to facilitate knowledge about chronic pain for adults: a systematic review with meta-analysis. Syst Rev. 2015. https://doi.org/10. 1186/s13643-015-0120-5.

Publisher's Note Springer Nature remains neutral with regard to jurisdictional claims in published maps and institutional affiliations. 